\title{
The $q_{T}$ subtraction method for top-quark production at hadron colliders
}

\author{
Roberto Bonciani $^{1,2}$, Stefano Catani ${ }^{3,4}$, Massimiliano Grazzini ${ }^{5, \mathrm{a}, \mathrm{b}}$, Hayk Sargsyan $^{5}$, Alessandro Torre $^{5}$ \\ ${ }^{1}$ Dipartimento di Fisica, Università di Roma "La Sapienza", 00185 Rome, Italy \\ ${ }^{2}$ INFN, Sezione di Roma, 00185 Rome, Italy \\ ${ }^{3}$ INFN, Sezione di Firenze, 50019 Sesto Fiorentino, Florence, Italy \\ ${ }^{4}$ Dipartimento di Fisica e Astronomia, Università di Firenze, 50019 Sesto Fiorentino, Florence, Italy \\ ${ }^{5}$ Physik-Institut, Universität Zürich, 8057 Zurich, Switzerland
}

Received: 25 August 2015 / Accepted: 13 November 2015 / Published online: 9 December 2015

(C) The Author(s) 2015. This article is published with open access at Springerlink.com

\begin{abstract}
We consider QCD radiative corrections to topquark pair production at hadron colliders. We use the $q_{T}$ subtraction formalism to perform a fully differential computation for this process. Our calculation is accurate up to the next-to-leading order in QCD perturbation theory and it includes all the flavour off-diagonal partonic channels at the next-to-next-to-leading order. We present a comparison of our numerical results with those obtained with the publicly available numerical programs MCFM and TOP++.

The top quark $(t)$ has a special role [1] in elementary particle physics. Being the heaviest known fundamental constituent, with a mass of about $173.3 \mathrm{GeV}$ [2], it couples strongly to the Higgs boson and it is crucial to the hierarchy problem. Within the Standard Model (SM) the main source of top-quark events in collisions at hadron colliders is top-quark pair production. Many New Physics (NP) models predict the existence of top partners with masses close to the electroweak symmetry breaking scale, which exhibit similar properties as the top quark and can decay into it. Studying the production of $t \bar{t}$ pairs at hadron colliders can not only shed light on the nature of the electroweak symmetry breaking but it also provides information on the backgrounds of many NP models.

The theoretical efforts for obtaining precision predictions for top-quark pair production at hadron colliders started almost three decades ago with the calculation of the nextto-leading order (NLO) QCD corrections to the total cross section [3-6] and kinematical distributions [7] for this production process. The NLO calculations of the total cross section of Refs. [3-6] were carried out numerically. The expres-
\end{abstract}

\footnotetext{
a e-mail: grazzini@physik.uzh.ch

${ }^{\mathrm{b}}$ On leave of absence from INFN, Sezione di Firenze, Sesto Fiorentino, Florence, Italy
}

sions in analytic form of the total partonic cross section ${ }^{1}$ at NLO were obtained in Ref. [11]. Recently the calculation of the next-to-next-to-leading order (NNLO) QCD corrections to the $t \bar{t}$ total cross section was completed [12-15]. Besides the total cross section, differential cross sections and more general kinematical distributions are of great importance for precision studies. For instance, the $t \bar{t}$ (forward-backward and charge) asymmetry has received much attention in recent years (see, e.g., Ref. [16]). The $t \bar{t}$ asymmetry, which is nonvanishing starting from the NLO level $[17,18]$, has recently been computed up to the NNLO level [19]. Other NNLO results on differential distributions are starting to appear [2023].

This letter is devoted to the NNLO (and NLO) QCD calculation of $t \bar{t}$ production. In particular, we present the results of the first NNLO application of the $q_{T}$ subtraction formalism [24] to the process of $t \bar{t}$ production in hadron collisions.

At the partonic level, the NNLO calculation of $t \bar{t}$ production requires the evaluation of tree-level contributions with two additional unresolved partons in the final state, of oneloop contributions with one unresolved parton and of purely virtual contributions. The required tree-level and one-loop scattering amplitudes are known and they are the same amplitudes that enter the NLO calculation of $t \bar{t}+$ jet $[25,26]$, the associated production of a $t \bar{t}$ pair and one jet. The purely virtual contributions depend on the two-loop scattering amplitudes and on the square of one-loop scattering amplitudes. The two-loop amplitude for $t \bar{t}$ production is partly known in analytic form [27-30] and its complete computation has been carried out numerically [31,32]. The square of one-loop scattering amplitudes is known [33-35].

\footnotetext{
${ }_{1}$ A parametrisation [8] of this analytic NLO result is implemented in the numerical programs HATHOR [9] and TOP++ [10].
} 
The implementation of the various scattering amplitudes in a complete NNLO calculation at the fully differential (exclusive) level is a highly non-trivial task because of the presence of infrared (IR) divergences at intermediate stages of the calculation. In particular, these divergences do not permit a straightforward implementation of numerical techniques. Various methods have been proposed and used to overcome these difficulties at the NNLO level. The formalisms of antenna subtraction [36-41] and colourful subtraction $[42,43]$ are more related to NNLO extensions of established NLO formulations [44-48] of the subtraction method. The Stripper formalism [49-51] is a combination of the subtraction method with numerical techniques based on sector decomposition [52,53]. Variants of the subtraction methods are the $q_{T}$ subtraction formalism [24] and the recently proposed $N$-jettiness subtraction [54-56].

The NNLO computations in Refs. [12-15,19] for $t \bar{t}$ production have been performed by using the Stripper method [49]. Parallely, an ongoing effort is being made by using the antenna subtraction method $[39,57]$, which led to the NNLO fully differential computation of $t \bar{t}$ production in the $q \bar{q}$ channel $[21,22,58]$ at leading colour and including the light-quark contributions.

The $q_{T}$ subtraction formalism [24] is a method to handle and cancel the IR divergences at the NLO and NNLO level. The method has been successfully applied to the fully differential computation of NNLO QCD corrections to several hard-scattering processes [24,59-67]. The method uses IR subtraction counterterms that are constructed by considering and explicitly computing the transverse-momentum $\left(q_{T}\right)$ distribution of the produced final-state system in the limit $q_{T} \rightarrow 0$. If the produced final-state system is composed of non-QCD (colourless) partons (e.g., leptons, vector bosons or Higgs bosons), the behaviour of the $q_{T}$ distribution in the limit $q_{T} \rightarrow 0$ has a universal (process-independent) structure that is explicitly known up to the NNLO level through the formalism of transverse-momentum resummation [68]. These results on transverse-momentum resummation are sufficient to fully specify the $q_{T}$ subtraction formalism for this entire class of processes. Therefore, up to now, the applications of the $q_{T}$ subtraction formalism have been limited to the production of colourless high-mass systems in hadron collisions. In this Letter we present first results on the application of the $q_{T}$ subtraction method to the NNLO computation of heavyquark production in hadron collisions. To this purpose, we use the recent progress on transverse-momentum resummation for heavy-quark production [69-71]. We exploit the formulation of transverse-momentum resummation in Ref. [71] that includes the complete dependence on the kinematics of the heavy-quark pair. This dependence and, in particular, the complete control on the heavy-quark azimuthal correlations are essential (see below) to extract all the NNLO counterterms of the $q_{T}$ subtraction method. Although the structure of transverse-momentum resummation for heavyquark production is fully worked out up to the NNLO level, the explicit NNLO results for the hard-virtual factors [71] in the flavour diagonal partonic channels $q \bar{q} \rightarrow t \bar{t}+X$ and $g g \rightarrow t \bar{t}+X$ ( $X$ denotes the unobserved inclusive final state) are not yet known. Therefore, in the NNLO calculation of this paper we present numerical results for all the flavour off-diagonal channels $a b \rightarrow t \bar{t}+X$, with $a b=q g(\bar{q} g), q q(\bar{q} \bar{q}), q q^{\prime}\left(\bar{q} \bar{q}^{\prime}\right), q \bar{q}^{\prime}\left(\bar{q} q^{\prime}\right)\left(q\right.$ and $q^{\prime}$ denote quarks with different flavour).

The differential cross section $\mathrm{d} \sigma^{t \bar{t}}$ for the inclusive production process $p p(p \bar{p}) \rightarrow t \bar{t}+X$ is computable by convoluting the corresponding partonic cross sections $\mathrm{d} \hat{\sigma}_{a b}^{t \bar{t}}$ of the various partonic channels with the parton distribution functions (PDFs) of the colliding hadrons $p p(p \bar{p})$. According to the $q_{T}$ subtraction method [24], the (N)NLO partonic cross section $\mathrm{d} \hat{\sigma}_{(\mathrm{N}) \mathrm{NLO}}^{t \bar{t}}$ can be written as

$\mathrm{d} \hat{\sigma}_{(\mathrm{N}) \mathrm{NLO}}^{t \bar{t}}=\mathcal{H}_{(\mathrm{N}) \mathrm{NLO}}^{t \bar{t}} \otimes \mathrm{d} \hat{\sigma}_{\mathrm{LO}}^{t \bar{t}}+\left[\mathrm{d} \hat{\sigma}_{(\mathrm{N}) \mathrm{LO}}^{t \bar{t}+\mathrm{jet}}-\mathrm{d} \hat{\sigma}_{(\mathrm{N}) \mathrm{NLO}}^{t \bar{t}, \mathrm{CT}}\right]$

where $\mathrm{d} \hat{\sigma}_{(\mathrm{N}) \mathrm{LO}}^{t \bar{t}+\text { iet }}$ is the $t \bar{t}+$ jet cross section at (N)LO accuracy. Applying Eq. (1) at NLO, the leading-order (LO) cross section $\mathrm{d} \sigma_{\mathrm{LO}}^{t \vec{t}+\text { jet }}$ can be directly obtained by integrating the corresponding tree-level scattering amplitudes. Applying Eq. (1) at NNLO, $\mathrm{d} \sigma_{\mathrm{NLO}}^{t \bar{t}+\mathrm{jet}}$ can be evaluated by using any available NLO method (e.g., Refs. [44-48]) to handle and cancel the corresponding IR divergences. Therefore, $\mathrm{d} \sigma_{(\mathrm{N}) \mathrm{LO}}^{t \bar{t}+\mathrm{jet}}$ is IR finite provided $q_{T} \neq 0$.

The square bracket term of Eq. (1) is IR finite in the limit $q_{T} \rightarrow 0$, but its individual contributions, $\mathrm{d} \sigma_{(\mathrm{N}) \mathrm{LO}}^{t \bar{t}+\mathrm{jet}}$ and $\mathrm{d} \sigma_{(\mathrm{N}) \mathrm{NLO}}^{t \bar{t}, \mathrm{CT}}$, are separately divergent. The IR subtraction counterterm $\mathrm{d} \sigma_{(\mathrm{N}) \mathrm{NLO}}^{t \bar{t}, \mathrm{CT}}$ is obtained from the (N)NLO perturbative expansion (see, e.g., Refs. [72,73]) of the resummation formula of the logarithmically enhanced contributions to the $q_{T}$ distribution of the $t \bar{t}$ pair [69-71]: the explicit form of $\mathrm{d} \sigma_{(\mathrm{N}) \mathrm{NLO}}^{t \bar{t}, \mathrm{CT}}$ can be completely worked out up to NNLO accuracy. For example, at the NLO, the explicit expression of $\mathrm{d} \hat{\sigma}_{\mathrm{NLO}}^{t \bar{t}, \mathrm{CT}}$ in the partonic channel $a b \rightarrow t \bar{t}+X$ is

$$
\begin{aligned}
\mathrm{d} \hat{\sigma}_{\mathrm{NLO} a b}^{t \bar{t}, \mathrm{CT}}= & \sum_{c=q, \bar{q}, g} \frac{\alpha_{\mathrm{S}}}{\pi}\left(\Sigma_{c \bar{c} \leftarrow a b}^{(1)}+\Sigma_{c \bar{c} \leftarrow a b}^{(1) t \bar{t}-\mathrm{new}}\right) \frac{\mathrm{d} q_{T}^{2}}{M^{2}} \\
& \otimes \mathrm{d} \hat{\sigma}_{\mathrm{LO} c \bar{c}}^{t \bar{t}},
\end{aligned}
$$

where $\alpha_{\mathrm{S}}$ is the QCD coupling, $M$ is the invariant mass of the produced $t \bar{t}$ pair and $\mathrm{d} \hat{\sigma}_{\mathrm{LO} a b}^{t \bar{t}}$ is the LO partonic cross section. The expression (2) involves convolutions (which are denoted by the symbol $\otimes$ ) with respect to the longitudinal-momentum fractions $z_{1}$ and $z_{2}$ of the colliding partons $c$ and $\bar{c}$ in $\mathrm{d} \hat{\sigma}_{\mathrm{LO}}^{t \bar{t}} \bar{c}$. The integration variable $q_{T}$ in Eq. (2) corresponds, in the limit $q_{T} \rightarrow 0$, to the transverse momentum of the produced $t \bar{t}$ pair 
in the cross section $\mathrm{d} \sigma_{\mathrm{LO}}^{t \bar{t}+\text { jet }}$ on the right-hand side of Eq. (1). The function $\Sigma_{c \bar{c} \leftarrow a b}^{(1)}$ enters into the $q_{T}$ subtraction method [24] for hard-scattering production of a generic final-state system. Its explicit form is [72,73]

$$
\begin{aligned}
\Sigma_{c \bar{c}}^{(1)} & \leftarrow a b \\
= & -\frac{1}{2} A_{c}^{(1)} \delta_{c a} \delta_{\bar{c} b} \delta\left(1-z_{2}\right) \delta\left(1-z_{2}\right) \tilde{I}_{2}\left(q_{T} / M\right) \\
& -\left[\delta_{c a} \delta_{\bar{c} b} \delta\left(1-z_{1}\right) \delta\left(1-z_{2}\right) B_{c}^{(1)}\right. \\
& \left.+\delta_{c a} \delta\left(1-z_{1}\right) P_{\bar{c} b}^{(1)}\left(z_{2}\right)+\delta_{\bar{c} b} \delta\left(1-z_{2}\right) P_{c a}^{(1)}\left(z_{1}\right)\right] \\
& \times \tilde{I}_{1}\left(q_{T} / M\right),
\end{aligned}
$$

and it derives from the small- $q_{T}$ singular behavior of the $q_{T}$ cross section for the production of a colourless system in the partonic $c \bar{c}$ production channel. The coefficients $A_{c}^{(1)}$ and $B_{c}^{(1)}$ are the first-order resummation coefficients for transverse-momentum resummation $\left(A_{q}^{(1)}=C_{F}, A_{g}^{(1)}=\right.$ $\left.C_{A}, B_{q}^{(1)}=-3 / 2 C_{F}, B_{g}^{(1)}=-\left(11 / 6 C_{A}-n_{F} / 3\right)\right)$. The functions $P_{a b}^{(1)}(z)$ are the lowest-order DGLAP kernels (the overall normalizazion is specified according to the notation in Eq. (41) of Ref. [72]). The functions $\tilde{I}_{k}\left(q_{T} / M\right)(k=1,2)$, which appear in Eq. (3), encapsulate the singular behavior at small $q_{T}$, and they are explicitly given in Appendix B of Ref. [72]. The other function $\Sigma_{c \bar{c} \leftarrow a b}^{(1) t \bar{t}-\text { new }}$ in the round-bracket factor of Eq. (2) is due to soft radiation and it is an additional term that is specific of the $q_{T}$ subtraction method for the case of heavy-quark pair production. This function reads

$$
\begin{aligned}
\Sigma_{c \bar{c} \leftarrow a b}^{(1) t \bar{t}-\text { new }}\left(z_{1}, z_{2} ; q_{T} / M\right) & \\
= & -\delta_{c a} \delta_{\bar{c} b} \delta\left(1-z_{1}\right) \delta\left(1-z_{2}\right) \\
& \times \frac{\left\langle\mathcal{M}_{c \bar{c} \rightarrow t \bar{t} \mid}\left(\Gamma_{t}^{(1)}+\Gamma_{t}^{(1) \dagger}\right) \mid \mathcal{M}_{c \bar{c} \rightarrow t \bar{t}}\right\rangle}{\left|\mathcal{M}_{c \bar{c} \rightarrow t \bar{t}}\right|^{2}} \tilde{I}_{1}\left(q_{T} / M\right),
\end{aligned}
$$

where $\Gamma_{t}^{(1)}$ is the first-order term of the soft anomalous dimension for transverse-momentum resummation in heavyquark production and its explicit expression is given in Eq. (33) of Ref. [71]. This soft anomalous dimension is a colour space matrix that acts onto the colour indices of the four partons $\{c, \bar{c}, t, \bar{t}\}$ in the Born level scattering amplitude $\left|\mathcal{M}_{c \bar{c} \rightarrow t \bar{t}}\right\rangle$ of the partonic process $c \bar{c} \rightarrow t \bar{t}$. The colour space notation is specified in Ref. [71] and, in particular, $\left|\mathcal{M}_{c \bar{c} \rightarrow t \bar{t}}\right|^{2}=\left\langle\mathcal{M}_{c \bar{c} \rightarrow t \bar{t}} \mid \mathcal{M}_{c \bar{c} \rightarrow t \bar{t}}\right\rangle$ denotes the colour summed square amplitude that contributes to $d \hat{\sigma}_{L}^{t \bar{t}} O c \bar{c}$, whereas the factor $\left\langle\mathcal{M}_{c \bar{c} \rightarrow t \bar{t}}\left|\left(\Gamma_{t}^{(1)}+\Gamma_{t}^{(1) \dagger}\right)\right| \mathcal{M}_{c \bar{c} \rightarrow t \bar{t}}\right\rangle$ embodies colour correlation terms with a definite kinematical dependence.

The first-order hard-collinear (IR finite) counterterms $\mathcal{H}_{\mathrm{NLO}}^{t \bar{t}}$ are also completely known [69-71] for all the partonic channels. The second-order (IR finite) counterterms
$\mathcal{H}_{\mathrm{NNLO}}^{t \bar{t}}$ are not yet fully known. However, $\mathcal{H}_{\mathrm{NNLO}}^{t \bar{t}}$ can be explicitly determined for all the flavour off-diagonal partonic channels. In these off-diagonal channels, $\mathcal{H}_{\mathrm{NNLO}}^{t \bar{t}}$ embodies process-dependent and process-independent contributions. The process-dependent contributions to $\mathcal{H}_{\mathrm{NNLO}}^{t \bar{t}}$ derive from the knowledge of the one-loop virtual amplitudes of the partonic processes $q \bar{q} \rightarrow t \bar{t}$ and $g g \rightarrow t \bar{t}$, and from the explicit results on the NLO azimuthal correlation terms in the transverse-momentum resummation formalism [71] (see, in particular, Eq. (25) in Ref. [71] and accompanying comments). The process-independent contributions to $\mathcal{H}_{\text {NNLO }}^{t \bar{t}}$ are analogous to those that contribute to Higgs boson [24] and vector boson [60] production, and they are explicitly known [68,74-78].

Having discussed the content of Eq. (1), we are in a position to apply it to $t \bar{t}$ production and to obtain the complete NLO results plus the NNLO corrections in all the flavour off-diagonal partonic channels. Our NLO implementation of the calculation has the main purpose of illustrating the applicability of the $q_{T}$ subtraction method to heavy-quark production and, in particular, of cross-checking the $q_{T}$ subtraction methodology by numerical comparisons with NLO calculations performed by using more established NLO methods. Our NNLO results on $t \bar{t}$ production represent a first step (due to the missing flavour diagonal partonic channels) towards the complete NNLO calculation for this production process. Up to NLO accuracy our numerical implementation is based on the scattering amplitudes and phase space generation of the MCFM program [79], suitably modified for $q_{T}$ subtraction along the lines of the corresponding numerical programs for Higgs boson [24] and vector boson [60] production. At NNLO accuracy the $t \bar{t}+$ jet cross section is evaluated by using the MUNICH code [80], which provides a fully automated implementation of the NLO dipole subtraction formalism [46-48] as well as an interface to the one-loop generator OPENLOOPS [81] to obtain all the required (spinand colour-correlated) tree-level and one-loop amplitudes. For the evaluation of tensor integrals we rely on the COLLIER library [82], which is based on the Denner-Dittmaier reduction techniques $[83,84]$ of tensor integrals and on the scalar integrals of Ref. [85]. In OPENLOOPS problematic phase space points are addressed with a rescue system that uses the quadruple-precision implementation of the OPP method in CUTTOOLs [86] with scalar integrals from ONELOOP [87].

We start the presentation of our results by considering pp collisions at $\sqrt{s}=8 \mathrm{TeV}$. We use the MSTW2008 [88] PDFs with the QCD running coupling $\alpha_{\mathrm{S}}$ evaluated at each corresponding order (i.e., we use $(n+1)$-loop $\alpha_{\mathrm{S}}$ at $\mathrm{N}^{n} \mathrm{LO}$, with $n=1,2$ ). The pole mass of the top quark is $m_{t}=$ $173.3 \mathrm{GeV}$. The renormalisation and factorisation scales, $\mu_{R}$ and $\mu_{F}$, are fixed at $\mu_{R}=\mu_{F}=m_{t}$.

In Figs. 1 and 2 we compare the NLO differential distributions obtained by using MCFM (which implements the dipole 


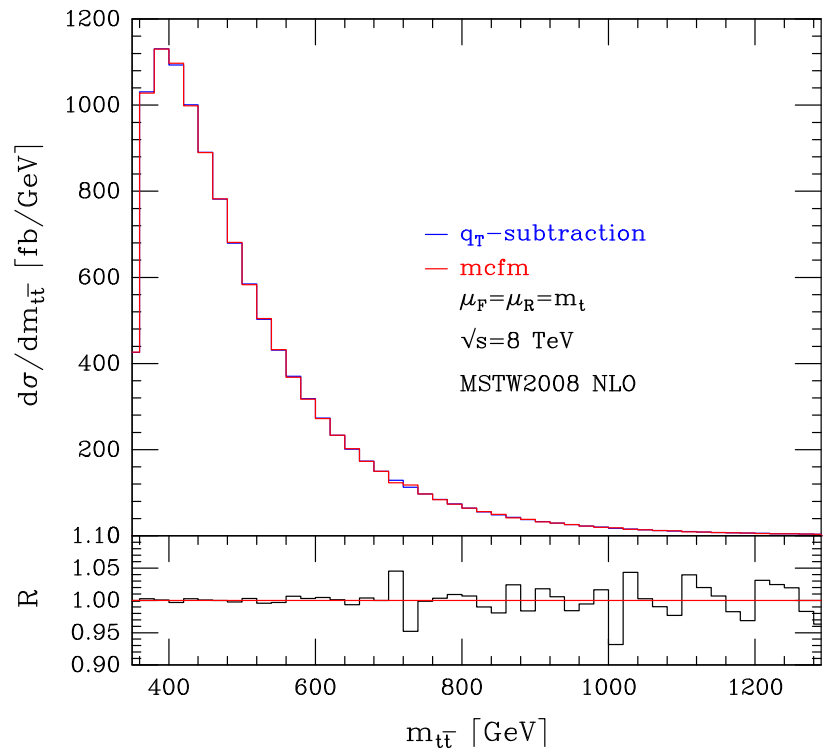

(a)

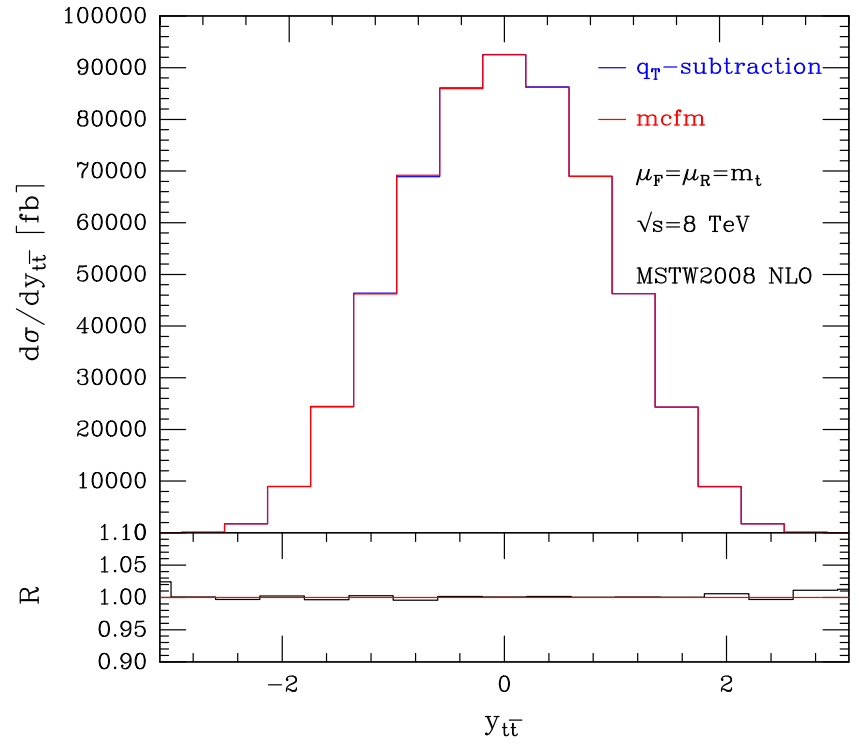

(b)

Fig. 1 The invariant mass (left) and rapidity (right) distributions of the $t \bar{t}$ pair at the LHC $(\sqrt{s}=8 \mathrm{TeV})$ computed at NLO accuracy. Comparison of our results (blue) with the MCFM results (red). The lower panel presents the ratio of our results over the MCFM results

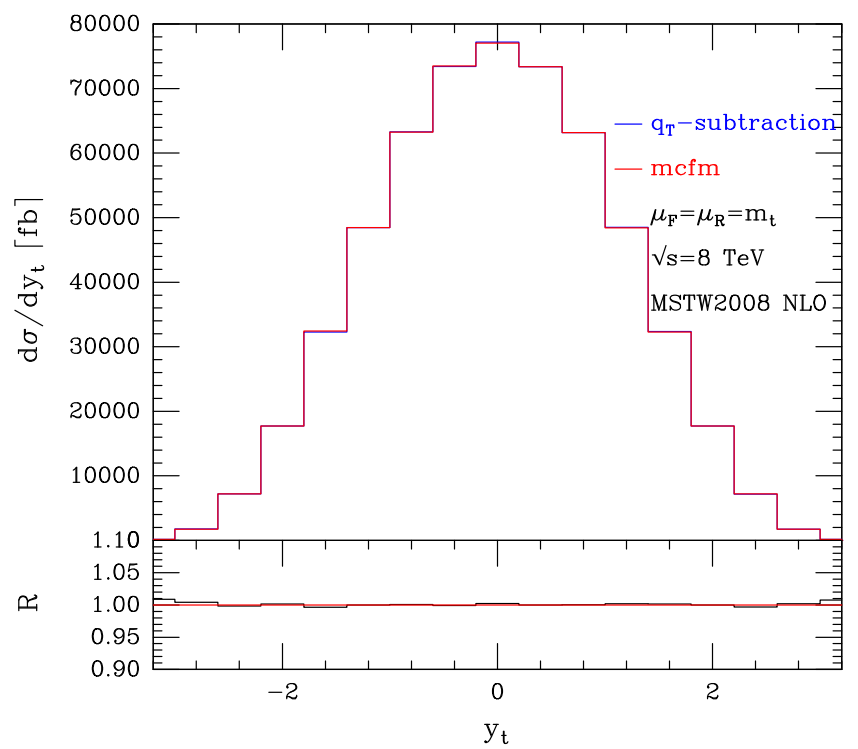

(a)

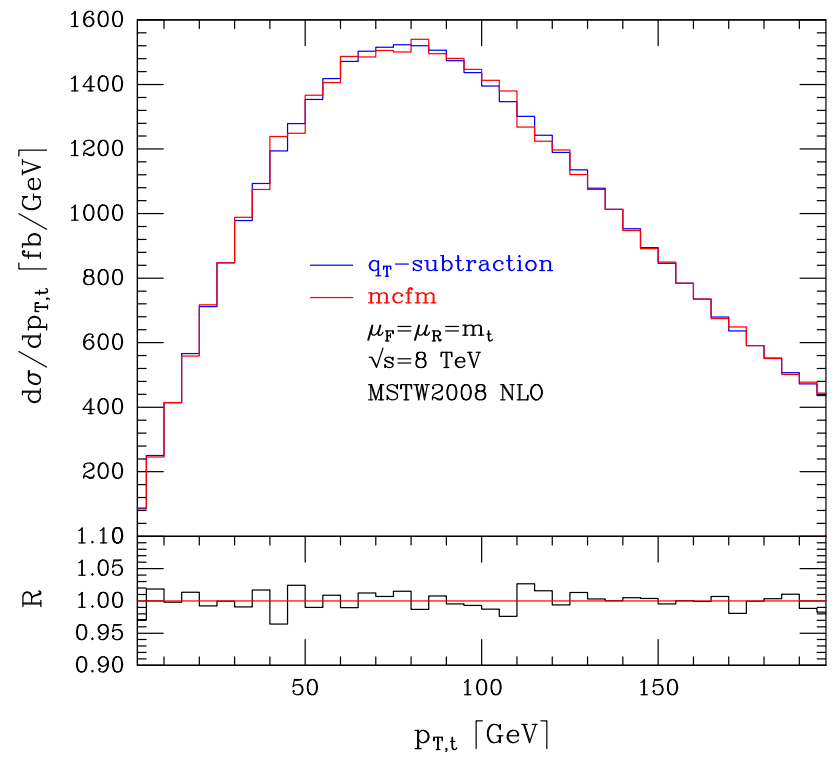

(b)

Fig. 2 The rapidity (left) and transverse-momentum (right) distributions of the top quark at the LHC $(\sqrt{s}=8 \mathrm{TeV})$ computed at NLO accuracy. Comparison of our results (blue) with the MCFM results (red). The lower panel presents the ratio of our results over the MCFM results

subtraction method [46-48]) with those obtained by using our numerical program. ${ }^{2}$ In particular in Fig. 1 we consider the invariant mass $\left(m_{t \bar{t}}\right)$ distribution (left) and the rapidity $\left(y_{t \bar{t}}\right)$ distribution (right) of the $t \bar{t}$ pair. In Fig. 2 we consider the

$\overline{2}$ These NLO results are obtained by using MCFM- v7.0 with about 9 hours of run on an Intel Xeon $2.4 \mathrm{Ghz}$, corresponding to a total of $2 \times 10^{6}$ integral evaluations. Our NLO implementation of $q_{T}$ subtraction requires about 30 times higher statistics to obtain comparable results and the corresponding runtime is a factor of 3 larger. rapidity $\left(y_{t}\right)$ distribution (left) and the transverse-momentum $\left(p_{T, t}\right)$ distribution (right) of the top quark. We clearly see that the distributions obtained with $q_{T}$ subtraction are in excellent agreement with those obtained with MCFM. We have checked that the agreement persists also for different choices of $\mu_{R}$ and $\mu_{F}$.

We now move to consider the NNLO contributions and, in particular, we compute the total cross section for $t \bar{t}$ production. In Table 1 we report our results and we compare 
Table 1 Total cross sections for $t \bar{t}$ production. NLO and (partial) NNLO results from $q_{T}$ subtraction compared with the corresponding results from TOP++ for $p p$ collisions at $\sqrt{s}=8 \mathrm{TeV}$

\begin{tabular}{llll}
\hline Cross section $[\mathrm{pb}]$ & NLO & $\left.\mathcal{O}\left(\alpha_{\mathrm{S}}^{4}\right)\right|_{q g}$ & $\left.\mathcal{O}\left(\alpha_{\mathrm{S}}^{4}\right)\right|_{q(\bar{q}) q^{\prime}}$ \\
\hline$q_{T}$ subtraction & $226.2(1)$ & $-2.25(5)$ & $0.151(3)$ \\
TOP++ & 226.3 & -2.253 & 0.148
\end{tabular}

Table 2 Total cross sections for $t \bar{t}$ production. NLO and (partial) NNLO results from $q_{T}$ subtraction compared with the corresponding results from ToP++ for $p \bar{p}$ collisions at $\sqrt{s}=2 \mathrm{TeV}$

\begin{tabular}{llll}
\hline Cross section $[\mathrm{fb}]$ & NLO & $\left.\mathcal{O}\left(\alpha_{\mathrm{S}}^{4}\right)\right|_{q g}$ & $\left.\mathcal{O}\left(\alpha_{\mathrm{S}}^{4}\right)\right|_{q(\bar{q}) q^{\prime}}$ \\
\hline$q_{T}$ subtraction & $7083(3)$ & $-61.5(5)$ & $1.33(1)$ \\
ToP++ & 7086 & -61.53 & 1.33 \\
\hline
\end{tabular}

them with the corresponding results from the numerical program TOP++ [10], which implements the NNLO calculation of Refs. [12-15]. Specifically, we report the complete NLO results and the $\mathcal{O}\left(\alpha_{\mathrm{S}}^{4}\right)$ contributions to the NNLO cross section from the flavour off-diagonal partonic channels $a b \rightarrow$ $t \bar{t}+X$. The contribution from all the channels with $a b=$ $q g, \bar{q} g$ is labelled by the subscript $q g$, and the contribution from all the channels with $a b=q q, \bar{q} \bar{q}, q q^{\prime}, \bar{q} \bar{q}^{\prime}, q \bar{q}^{\prime}, \bar{q} q^{\prime}$ is labelled by the subscript $q(\bar{q}) q^{\prime}$. From Table 1 we see that the results obtained by using $q_{T}$ subtraction are in agreement with those from TOP++. We note that the numerical uncertainties of our $\mathcal{O}\left(\alpha_{\mathrm{S}}^{4}\right)$ results are at the $2 \%$ level. This is due to the fact that in both the $q g$ and the $q(\bar{q}) q^{\prime}$ channels at $\sqrt{s}=8 \mathrm{TeV}$ there is a strong quantitative cancellation between the contributions of the two terms in the right-hand side of Eq. (1) (the term that is proportional to $\mathcal{H}_{\mathrm{NNLO}}^{t \bar{t}}$ and the term in the square bracket). The numerical uncertainties of our $\mathcal{O}\left(\alpha_{\mathrm{S}}^{4}\right)$ calculation can be reduced by considering different centre-of-mass energies. In particular, the numerical cancellation that we have mentioned is reduced by decreasing the centre-of-mass energy of the collision.

We have computed the total cross section for $p \bar{p}$ collisions at $\sqrt{s}=2 \mathrm{TeV}$ and we report the corresponding results in Table 2. We note that the numerical agreement between our calculation and the TOP++ result is still satisfactory, and the numerical uncertainties of our $\mathcal{O}\left(\alpha_{\mathrm{S}}^{4}\right)$ results are reduced below the $1 \%$ level.

Using TOP++ we can compute the complete NNLO result and we note that the flavour off-diagonal partonic channels contribute to about $10 \%$ of the total result at $\mathcal{O}\left(\alpha_{\mathrm{S}}^{4}\right)$ for both collision energies considered in Tables 1 and 2.

We have presented the first application of the $q_{T}$ subtraction method to top-quark pair production at hadron colliders. Our implementation is based on the formulation of transverse-momentum resummation for heavy-quark production of Ref. [71], which includes the complete depen- dence on the kinematics of the heavy-quark pair. Our computation is accurate at NLO in QCD perturbation theory and it includes all the off-diagonal partonic channels at NNLO accuracy. At NLO we have compared our results for various kinematical distributions with those obtained by using the MCFM program, and we find good agreement. At NNLO our results for the $t \bar{t}$ total cross section agree with the corresponding results obtained by using the TOP++ program. The extension of our NNLO computation to include the missing $q \bar{q} \rightarrow t \bar{t}+X$ and $g g \rightarrow t \bar{t}+X$ channels requires the evaluation of the second-order hard-collinear functions $\mathcal{H}_{\mathrm{NNLO}}^{t \bar{t}}$ [71], and an implementation of the two-loop virtual amplitudes, which, at present, are known only in numerical form [31,32].

The computation that we have performed in this paper can straightforwardly be extended to the production of massivequark pairs of different flavour (e.g. bottom-quark pair). The extension of the method to production processes with massless coloured particles in the final state (e.g. inclusive dijet production) is definitely non-trivial and it would require additional theoretical advancements.

Acknowledgments We are grateful to Stefan Kallweit for his help with the MUNICH code. This research was supported in part by the Swiss National Science Foundation (SNF) under contract 200021-156585 and by the Research Executive Agency (REA) of the European Union under the Grant Agreement number PITN-GA-2012-316704 (Higgstools). The work of RB was partly supported by the European Community Seventh Framework Programme FP7/2007-2013, under Grant Agreement N.302997. We acknowledge the hospitality and partial support of the Galileo Galilei Institute (GGI) in Florence, where part of this work has been carried out during the Workshop Prospects and Precision at the Large Hadron Collider at $14 \mathrm{TeV}$.

Open Access This article is distributed under the terms of the Creative Commons Attribution 4.0 International License (http://creativecomm ons.org/licenses/by/4.0/), which permits unrestricted use, distribution, and reproduction in any medium, provided you give appropriate credit to the original author(s) and the source, provide a link to the Creative Commons license, and indicate if changes were made. Funded by SCOAP $^{3}$.

\section{References}

1. K.A. Olive et al. Particle Data Group Collaboration, Chin. Phys. C 38, 090001 (2014)

2. LHC/Tevatron Note, ATLAS and CDF and CMS and D0 Collaborations, arXiv:1403.4427 [hep-ex]

3. P. Nason, S. Dawson, R.K. Ellis, Nucl. Phys. B 303, 607 (1988)

4. W. Beenakker, H. Kuijf, W.L. van Neerven, J. Smith, Phys. Rev. D 40, 54 (1989)

5. W. Beenakker, W.L. van Neerven, R. Meng, G.A. Schuler, J. Smith, Nucl. Phys. B 351, 507 (1991)

6. P. Nason, S. Dawson, R. K. Ellis, Nucl. Phys. B 327, 49 (1989). [Erratum Nucl. Phys. B 335 (1990) 260]

7. M.L. Mangano, P. Nason, G. Ridolfi, Nucl. Phys. B 373, 295 (1992)

8. U. Langenfeld, S. Moch, P. Uwer, Phys. Rev. D 80, 054009 (2009). arXiv:0906.5273 [hep-ph] 
9. M. Aliev, H. Lacker, U. Langenfeld, S. Moch, P. Uwer, M. Wiedermann, Comput. Phys. Commun. 182, 1034 (2011). arXiv:1007.1327 [hep-ph]

10. M. Czakon, A. Mitov, Comput. Phys. Commun. 185, 2930 (2014). arXiv:1112.5675 [hep-ph]

11. M. Czakon, A. Mitov, Nucl. Phys. B 824, 111 (2010). arXiv:0811.4119 [hep-ph]

12. P. Bärnreuther, M. Czakon, A. Mitov, Phys. Rev. Lett. 109, 132001 (2012). arXiv:1204.5201 [hep-ph]

13. M. Czakon, A. Mitov, JHEP 1212, 054 (2012). arXiv:1207.0236 [hep-ph]

14. M. Czakon, A. Mitov, JHEP 1301, 080 (2013). arXiv:1210.6832 [hep-ph]

15. M. Czakon, P. Fiedler, A. Mitov, Phys. Rev. Lett. 110, 252004 (2013). arXiv:1303.6254 [hep-ph]

16. J.H. Kühn, G. Rodrigo, arXiv: 1411.4675 [hep-ph]

17. J.H. Kühn, G. Rodrigo, Phys. Rev. D 59, 054017 (1999). arXiv:hep-ph/9807420

18. J.H. Kühn, G. Rodrigo, Phys. Rev. Lett. 81, 49 (1998). arXiv:hep-ph/9802268

19. M. Czakon, P. Fiedler, A. Mitov, Phys. Rev. Lett. 115(5), 052001 (2015). arXiv:1411.3007 [hep-ph]

20. M. Czakon, A. Mitov, J. Rojo, arXiv:1501.01112 [hep-ph]

21. G. Abelof, A. Gehrmann-De Ridder, JHEP 1412, 076 (2014). arXiv: 1409.3148 [hep-ph]

22. G. Abelof, A.G.D. Ridder, I. Majer, arXiv:1506.04037 [hep-ph]

23. M. Czakon, talk given at Radcor-Loopfest 2015, Los Angeles (2015)

24. S. Catani, M. Grazzini, Phys. Rev. Lett. 98, 222002 (2007). arXiv:hep-ph/0703012

25. S. Dittmaier, P. Uwer, S. Weinzierl, Phys. Rev. Lett. 98, 262002 (2007). arXiv:hep-ph/0703120 [HEP-PH]

26. S. Dittmaier, P. Uwer, S. Weinzierl, Eur. Phys. J. C 59, 625 (2009). arXiv:0810.0452 [hep-ph]

27. R. Bonciani, A. Ferroglia, T. Gehrmann, D. Maitre, C. Studerus, JHEP 0807, 129 (2008). arXiv:0806.2301 [hep-ph]

28. R. Bonciani, A. Ferroglia, T. Gehrmann, C. Studerus, JHEP 0908 , 067 (2009). arXiv:0906.3671 [hep-ph]

29. R. Bonciani, A. Ferroglia, T. Gehrmann, A. von Manteuffel, C. Studerus, JHEP 1101, 102 (2011). arXiv:1011.6661 [hep-ph]

30. R. Bonciani, A. Ferroglia, T. Gehrmann, A. von Manteuffel, C. Studerus, JHEP 1312, 038 (2013). arXiv: 1309.4450 [hep-ph]

31. M. Czakon, Phys. Lett. B 664, 307 (2008). arXiv:0803.1400 [hep$\mathrm{ph}]$

32. P. Bärnreuther, M. Czakon, P. Fiedler, JHEP 1402, 078 (2014). arXiv:1312.6279 [hep-ph]

33. J.G. Korner, Z. Merebashvili, M. Rogal, Phys. Rev. D 77, 094011 (2008). [Erratum Phys. Rev. D 85 (2012) 119904]. arXiv:0802.0106 [hep-ph]

34. C. Anastasiou, S.M. Aybat, Phys. Rev. D 78, 114006 (2008). arXiv:0809.1355 [hep-ph]

35. B. Kniehl, Z. Merebashvili, J.G. Korner, M. Rogal, Phys. Rev. D 78, 094013 (2008). arXiv:0809.3980 [hep-ph]

36. D.A. Kosower, Phys. Rev. D 57, 5410 (1998). arXiv:hep-ph/9710213

37. D.A. Kosower, Phys. Rev. D 67, 116003 (2003). arXiv:hep-ph/0212097

38. D.A. Kosower, Phys. Rev. D 71, 045016 (2005). arXiv:hep-ph/0311272

39. A. Gehrmann-De Ridder, T. Gehrmann, E.W.N. Glover, JHEP 0509, 056 (2005). arXiv:hep-ph/0505111

40. A. Daleo, T. Gehrmann, D. Maitre, JHEP 0704, 016 (2007). arXiv:hep-ph/0612257

41. J. Currie, E.W.N. Glover, S. Wells, JHEP 1304, 066 (2013). arXiv:1301.4693 [hep-ph]
42. G. Somogyi, Z. Trocsanyi, V. Del Duca, JHEP 0506, 024 (2005). arXiv:hep-ph/0502226

43. V. Del Duca, C. Duhr, G. Somogyi, F. Tramontano, Z. Trocsanyi, JHEP 1504, 036 (2015). arXiv: 1501.07226 [hep-ph]

44. S. Frixione, Z. Kunszt, A. Signer, Nucl. Phys. B 467, 399 (1996). arXiv:hep-ph/9512328

45. S. Frixione, Nucl. Phys. B 507, 295 (1997). arXiv:hep-ph/9706545

46. S. Catani, M.H. Seymour, Phys. Lett. B 378, 287 (1996). arXiv:hep-ph/9602277

47. S. Catani, M.H. Seymour, Nucl. Phys. B 485, 291 (1997). [Erratumibid. B 510 (1998) 503]. arXiv:hep-ph/9605323

48. S. Catani, S. Dittmaier, M.H. Seymour, Z. Trocsanyi, Nucl. Phys. B 627, 189 (2002). arXiv:hep-ph/0201036

49. M. Czakon, Phys. Lett. B 693, 259 (2010). arXiv:1005.0274 [hep$\mathrm{ph}]$

50. M. Czakon, Nucl. Phys. B 849, 250 (2011). arXiv:1101.0642 [hep$\mathrm{ph}]$

51. M. Czakon, D. Heymes, Nucl. Phys. B 890, 152 (2014). arXiv: 1408.2500 [hep-ph]

52. T. Binoth, G. Heinrich, Nucl. Phys. B 585, 741 (2000). arXiv:hep-ph/0004013

53. C. Anastasiou, K. Melnikov, F. Petriello, Phys. Rev. D 69, 076010 (2004). arXiv:hep-ph/0311311

54. R. Boughezal, C. Focke, X. Liu, F. Petriello, arXiv:1504.02131 [hep-ph]

55. R. Boughezal, X. Liu, F. Petriello, Phys. Rev. D 91(9), 094035 (2015). arXiv:1504.02540 [hep-ph]

56. J. Gaunt, M. Stahlhofen, F. J. Tackmann, J. R. Walsh, arXiv: 1505.04794 [hep-ph]

57. G. Abelof, A. Gehrmann-De Ridder, JHEP 1104, 063 (2011). arXiv:1102.2443 [hep-ph]

58. G. Abelof, A. Gehrmann-De Ridder, P. Maierhofer, S. Pozzorini, JHEP 1408, 035 (2014). arXiv:1404.6493 [hep-ph]

59. M. Grazzini, JHEP 0802, 043 (2008). arXiv:0801.3232 [hep-ph]

60. S. Catani, L. Cieri, G. Ferrera, D. de Florian, M. Grazzini, Phys. Rev. Lett. 103, 082001 (2009). arXiv:0903.2120 [hep-ph]

61. G. Ferrera, M. Grazzini, F. Tramontano, Phys. Rev. Lett. 107, 152003 (2011). arXiv:1107.1164 [hep-ph]

62. G. Ferrera, M. Grazzini, F. Tramontano, Phys. Lett. B 740, 51 (2015). arXiv:1407.4747 [hep-ph]

63. S. Catani, L. Cieri, D. de Florian, G. Ferrera, M. Grazzini, Phys. Rev. Lett. 108, 072001 (2012). arXiv:1110.2375 [hep-ph]

64. M. Grazzini, S. Kallweit, D. Rathlev, A. Torre, Phys. Lett. B 731, 204 (2014). arXiv:1309.7000 [hep-ph]

65. F. Cascioli, T. Gehrmann, M. Grazzini, S. Kallweit, P. Maierhöfer, A. von Manteuffel, S. Pozzorini, D. Rathlev, L. Tancredi, E. Weihs, Phys. Lett. B 735, 311 (2014). arXiv:1405.2219 [hep-ph]

66. T. Gehrmann, M. Grazzini, S. Kallweit, P. Maierhöfer, A. von Manteuffel, S. Pozzorini, D. Rathlev, L. Tancredi, Phys. Rev. Lett. 113(21), 212001 (2014). arXiv:1408.5243 [hep-ph]

67. M. Grazzini, S. Kallweit, D. Rathlev, arXiv:1504.01330 [hep-ph]

68. S. Catani, L. Cieri, D. de Florian, G. Ferrera, M. Grazzini, Nucl. Phys. B 881, 414 (2014). arXiv:1311.1654 [hep-ph]

69. H.X. Zhu, C.S. Li, H.T. Li, D.Y. Shao, L.L. Yang, Phys. Rev. Lett. 110(8), 082001 (2013). arXiv:1208.5774 [hep-ph]

70. H.T. Li, C.S. Li, D.Y. Shao, L.L. Yang, H.X. Zhu, Phys. Rev. D 88, 074004 (2013). arXiv:1307.2464

71. S. Catani, M. Grazzini, A. Torre, Nucl. Phys. B 890, 518 (2014). arXiv: 1408.4564 [hep-ph]

72. G. Bozzi, S. Catani, D. de Florian, M. Grazzini, Nucl. Phys. B 737, 73 (2006). arXiv:hep-ph/0508068

73. G. Bozzi, S. Catani, D. de Florian, M. Grazzini, Nucl. Phys. B 791, 1 (2008). arXiv:0705.3887 [hep-ph]

74. S. Catani, M. Grazzini, Eur. Phys. J. C 72, 2013 (2012)

75. S. Catani, M. Grazzini, Eur. Phys. J. C 72, 2132 (2012). arXiv:1106.4652 [hep-ph] 
76. S. Catani, L. Cieri, D. de Florian, G. Ferrera, M. Grazzini, Eur. Phys. J. C 72, 2195 (2012). arXiv:1209.0158 [hep-ph]

77. T. Gehrmann, T. Lubbert, L.L. Yang, Phys. Rev. Lett. 109, 242003 (2012). arXiv:1209.0682 [hep-ph]

78. T. Gehrmann, T. Lubbert, L.L. Yang, JHEP 1406, 155 (2014). arXiv:1403.6451 [hep-ph]

79. J.M. Campbell, R.K. Ellis, W.T. Giele, Eur. Phys. J. C 75(6), 246 (2015). arXiv:1503.06182 [physics.comp-ph]

80. S. Kallweit, MUNICH is the abbreviation of "MUlti-chaNnel Integrator at Swiss $(\mathrm{CH})$ precision"- an automated parton level NLO generator (In preparation)

81. F. Cascioli, P. Maierhöfer, S. Pozzorini, Phys. Rev. Lett. 108, 111601 (2012). arXiv:1111.5206 [hep-ph]

82. A. Denner, S. Dittmaier, L. Hofer, PoS LL 2014, 071 (2014). arXiv:1407.0087 [hep-ph]
83. A. Denner, S. Dittmaier, Nucl. Phys. B 658, 175 (2003). arXiv:hep-ph/0212259

84. A. Denner, S. Dittmaier, Nucl. Phys. B 734, 62 (2006). arXiv:hep-ph/0509141

85. A. Denner, S. Dittmaier, Nucl. Phys. B 844, 199 (2011). arXiv: 1005.2076 [hep-ph]

86. G. Ossola, C.G. Papadopoulos, R. Pittau, JHEP 0803, 042 (2008). arXiv:00711.3596 [hep-ph]

87. A. van Hameren, Comput. Phys. Commun. 182, 2427 (2011). arXiv:1007.4716 [hep-ph]

88. A.D. Martin, W.J. Stirling, R.S. Thorne, G. Watt, Eur. Phys. J. C 63, 189 (2009). arXiv:0901.0002 [hep-ph] 